\section{Protein-bound molybdenum cofactor is bioavailable and rescues molybdenum cofactor-deficient $C$. elegans}

\author{
Kurt Warnhoff, 1,2,4 Thomas W. Hercher, ${ }^{3,4}$ \\ Ralf R. Mendel, ${ }^{3}$ and Gary Ruvkun ${ }^{1,2}$ \\ ${ }^{1}$ Department of Molecular Biology, Massachusetts General \\ Hospital, Boston, Massachusetts 02114; ${ }^{2}$ Department of \\ Genetics, Harvard Medical School, Boston, Massachusetts 02115; \\ ${ }^{3}$ Braunschweig University of Technology, 38106 Braunschweig, \\ Germany
}

The molybdenum cofactor (Moco) is a 520-Da prosthetic group that is synthesized in all domains of life. In animals, four oxidases (among them sulfite oxidase) use Moco as a prosthetic group. Moco is essential in animals; humans with mutations in genes that encode Moco biosynthetic enzymes display lethal neurological and developmental defects. Moco supplementation seems a logical therapy; however, the instability of Moco has precluded biochemical and cell biological studies of Moco transport and bioavailability. The nematode Caenorhabditis elegans can take up Moco from its bacterial diet and transport it to cells and tissues that express Moco-requiring enzymes, suggesting a system for Moco uptake and distribution. Here we show that protein-bound Moco is the stable, bioavailable species of Moco taken up by C. elegans from its diet and is an effective dietary supplement, rescuing a C. elegans model of Moco deficiency. We demonstrate that diverse Moco:protein complexes are stable and bioavailable, suggesting a new strategy for the production and delivery of therapeutically active Moco to treat human Moco deficiency.

Supplemental material is available for this article.

Received October 19, 2020; revised version accepted

December 1, 2020.

The molybdenum cofactor (Moco) is an ancient coenzyme that was present in the last universal common ancestor and that continues to be synthesized in all domains of life (Zhang and Gladyshev 2008; Weiss et al. 2016). Moco is a pterin-based organic prosthetic group that is comprised of a C6-substituted pyrano ring, a terminal phosphate, and a dithiolate group binding to molybdenum (Fig. 1A; Rajagopalan and Johnson 1992). In humans and other animals, Moco is required for the activity of four enzymes: sulfite oxidase, xanthine oxidase, aldehyde oxidase, and mitochondrial amidoxime reducing component (Schwarz et al. 2009). There are two forms of

\footnotetext{
[Keywords: molybdenum cofactor; sulfite oxidase; C. elegans; E. coli] ${ }^{4}$ These authors contributed equally to this work. Corresponding authors: ruvkun@molbio.mgh.harvard.edu, r.mendel@tu-bs.de

Article published online ahead of print. Article and publication date are online at http://www.genesdev.org/cgi/doi/10.1101/gad.345579.120.
}

eukaryotic Moco: the sulfite oxidase form and the xanthine oxidase form (Fig. 1A). These Moco species differ in the third Mo-S ligand, which is provided either by an enzyme-derived cysteine residue (sulfite oxidase form) or an inorganic sulfur (xanthine oxidase form) (Schwarz et al. 2009). The xanthine oxidase form of Moco is synthesized from the sulfite oxidase form via the enzyme Moco sulfurase (Fig. 1A; Bittner et al. 2001).

Both forms of Moco are synthesized by a highly conserved biosynthetic pathway (Fig. 1A; Mendel 2013). The genes necessary for Moco biosynthesis were first elucidated by genetic studies of chlorate resistance in bacteria (MacGregor 1975). The importance of Moco biosynthesis to human health is highlighted by Moco deficiency (MoCD), a rare inborn error of metabolism. MoCD is caused by loss-of-function mutations in genes encoding any of the human Moco biosynthetic enzymes and results in severe neurological dysfunction and neonatal lethality (Reiss and Hahnewald 2011; Huijmans et al. 2017). MoCD patients with mutations in MOCS1 (orthologous to bacterial moaA and moaC) can be treated with cyclic pyranopterin monophosphate (cPMP), a stable intermediate in Moco biosynthesis immediately downstream from MOCS1 (Veldman et al. 2010). However, cPMP treatment is not effective for patients with mutations in any of the downstream Moco biosynthetic enzymes. Purification and delivery of mature Moco would be an ideal therapeutic strategy for treating all forms of MoCD; however, free Moco is too unstable and oxygen-sensitive to be purified and therapeutically administered (Johnson et al. 1980; Mendel 1983). Furthermore, it is unclear whether mature Moco can cross cellular membranes.

Genetic evidence demonstrates that the nematode C. elegans retrieves Moco as well as cPMP from its bacterial diet (Warnhoff and Ruvkun 2019). However, nothing was known about the biochemical mechanism of Moco transfer between these two highly divergent organisms. Here we propose that Moco bound to protein is the stable and bioavailable Moco species that is harvested by C. elegans. We demonstrate that supplementation of purified protein-bound Moco rescues the lethality of Moco-deficient C. elegans feeding on Moco-deficient E. coli. We show that Moco bound to diverse Moco-containing proteins originating from bacteria, algae, fungi, and mammals, is bioavailable to C. elegans, and that this supplementation does not require Moco biosynthetic enzymes in C. elegans or its bacterial diet. This work suggests future mammalian therapeutic studies of supplemental protein-bound Moco and highlights the existence of a pathway for Moco transport.

\section{Results and Discussion}

\section{C. elegans acquires Moco from dietary E. coli}

Due to its instability, Moco has long been thought to be synthesized and used cell autonomously. So far only

(C) 2021 Warnhoff et al. This article is distributed exclusively by Cold Spring Harbor Laboratory Press for the first six months after the full-issue publication date (see http://genesdev.cshlp.org/site/misc/terms.xhtml). After six months, it is available under a Creative Commons License (Attribution-NonCommercial 4.0 International), as described at http://creativecommons.org/licenses/by-nc/4.0/. 


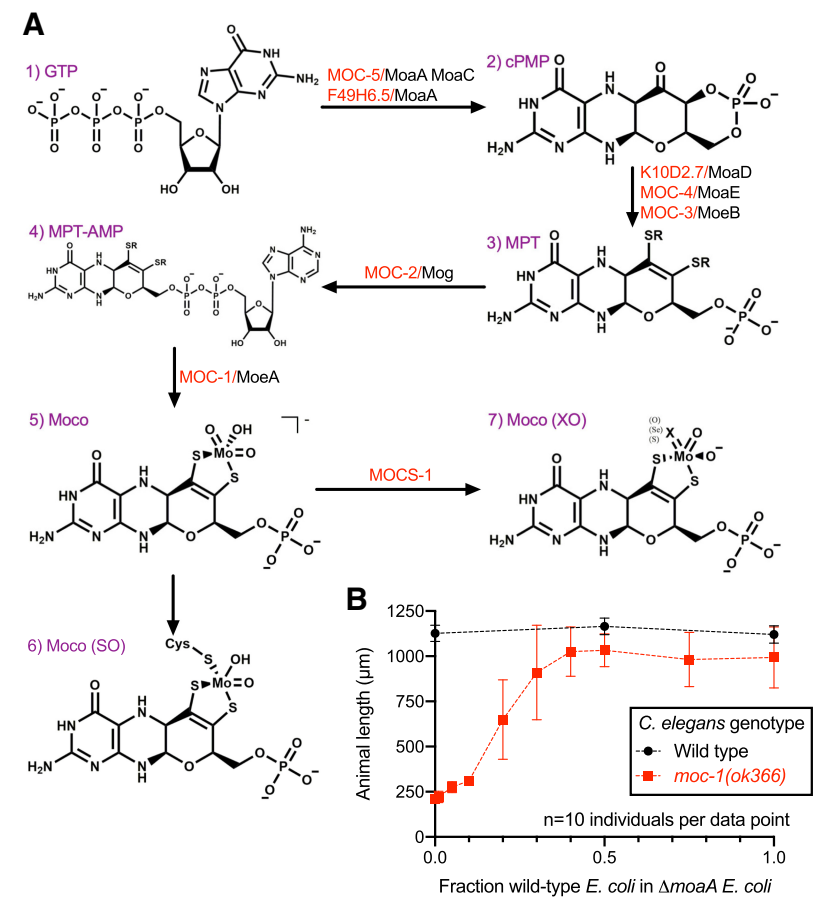

Figure 1. C. elegans acquires Moco from dietary E. coli. (A) C. elegans Moco biosynthesis pathway (red) and orthologous enzymes in E. coli (black) are displayed. Moco and its biosynthetic intermediates are displayed (purple): GTP is guanosine triphosphate (1), cPMP is cyclic pyranopterin monophosphate (2), MPT is molybdopterin (3), MPT-AMP is MPT-adenine monophosphate (4), Moco is the molybdenum cofactor (5), Moco (SO) is the sulfite oxidase form of the molybdenum cofactor $(6)$, and Moco $(\mathrm{XO})$ is the xanthine oxidase form of the molybdenum cofactor (7). C. elegans Moco sulfurase (MOCS1) has no clear homolog in $E$. coli, although $x d h C$ is the likely functional analog (Neumann et al. 2007). (B) Wild-type and moc-1 (ok366) C. elegans were synchronized at the Ll stage and cultured on mixtures of wild-type E. coli (synthesizes Moco) and $\triangle m o a A$ E. coli (cannot synthesize Moco) for $72 \mathrm{~h}$. The $Y$-axis shows animal length $(\mu \mathrm{m})$, where $1000 \mu \mathrm{m}$ roughly corresponds to fertile adulthood and $250 \mu \mathrm{m}$ roughly corresponds to the L1 stage. Average and standard deviation are displayed for each condition analyzed. Sample size $(n)$ was 10 individual animals assayed for each condition.

C. elegans has been described to have two pathways by which it can obtain Moco: endogenous Moco biosynthesis from GTP or dietary uptake of Moco (Warnhoff and Ruvkun 2019). Moco biosynthetic enzymes are conserved in all domains of life; in C. elegans these enzymes are encoded by the moc genes that mediate sequential steps in Moco biosynthesis (Fig. 1A). Using mutations in the C. elegans moc genes [i.e., the moc-1(ok366) null mutation] Moco biosynthesis can be interrupted in all cells. In the laboratory, $C$. elegans feed on a monoculture of $E$. coli. Thus, we can also use mutations in any of the genes of the $E$. coli Moco biosynthetic pathway to eliminate dietary Moco (i.e., the $\triangle m o a A$-null mutation). Either endogenous Moco synthesis in C. elegans or Moco produced by the diet $E$. coli and then consumed by $C$. elegans can support growth, development, and reproduction of $C$. elegans. However, when $C$. elegans cannot synthesize their own Moco and cannot obtain Moco from their diet, they arrest larval development and die due to inactivity of sulfite oxidase, the key Moco-using enzyme in animals (Warnhoff and Ruvkun 2019).
To test how much wild-type, Moco-producing bacteria was required to support growth and development of C. elegans defective in Moco biosynthesis, we mixed wild-type and $\triangle m o a A$ mutant $E$. coli at various ratios and tested for the ability of these mixtures to support the viability of moc-1 mutant $C$. elegans. We found that a substantial fraction (about $30 \%$ ) of the E. coli diet needed to be wild type (Moco producing) to support growth and development of moc-1 mutant animals (Fig. 1B).

\section{Moco bound to diverse proteins is taken up and used by C. elegans}

We hypothesized that $C$. elegans harvest bacterial Moco that is bound within the E. coli Moco-using enzymes. E. coli YiiM (EcYiiM) is one such Moco-using enzyme and mediates the reduction of $\mathrm{N}$-hydroxylated substrates (Kozmin et al. 2008; Namgung et al. 2018). To test whether Moco bound to EcYiiM can be absorbed by $C$. elegans, we purified recombinant EcYiiM protein from E. coli and used it to supplement the diet of Moco biosynthetic mutant $C$. elegans feeding on Moco-deficient $E$. coli, growth conditions that would otherwise result in $100 \%$ larval arrest and death. Consistent with the model that $C$. elegans harvests Moco from E. coli Moco-using enzymes, moc-1 mutant animals grown on Moco-deficient $E$. coli grew and developed well when their diet was supplemented with EcYiiM-bound Moco. (Fig. 2A, B). Thus, EcYiiM-bound Moco is bioavailable and can support the viability of otherwise Moco-deficient $C$. elegans.

To test whether the ability of $C$. elegans to harvest Moco from protein was more general to other Mocobinding proteins, we recombinantly expressed and purified two additional Moco-binding proteins in E. coli; nitrate reductase from the red bread mold Neurospora crassa (NcNR) and Moco-carrier protein from the green algae Volvox carteri (VcMCP) (Witte et al. 1998; Fischer et al. 2005; Hercher et al. 2020b). We also used the commercially available Moco-using enzyme xanthine oxidase (BtXO) purified from bovine milk (Enroth et al. 2000). Each Moco-binding protein was supplemented to moc-1 mutant $C$. elegans fed $\triangle$ moaA mutant $E$. coli. Similar to EcYiiM, supplementation with Moco bound to either $V c M C P$ or $N c N R$ supported the growth of moc-1 mutant $C$. elegans in the absence of any other dietary Moco (Fig. 2). To a lesser extent, BtXO supplementation also supported the growth of moc-1 mutant animals cultured on Moco-deficient E. coli (Fig. 2A,B). A possible explanation for the reduced efficacy of supplemental BtXO compared with EcYiiM, $V c M C P$, or $N c N R$ might be the form of Moco that is bound by these proteins. BtXO binds the xanthine oxidase form of Moco, while EcYiiM, VcMCP, and NcNR bind the sulfite oxidase form of Moco (Fig. 1A; Fischer et al. 2005; Hille et al. 2014; Namgung et al. 2018; Hercher et al. 2020b). In C. elegans and other animals, sulfite oxidase (SUOX1 ) is the key Moco-requiring enzyme necessary for viability; suox-1 null mutant animals arrest development similar to Moco-deficient animals (Warnhoff and Ruvkun 2019). We speculate that supplementation with the sulfite oxidase form of Moco can supply the appropriate Moco to support C. elegans SUOX-1 activity, whereas the supplementation with the xanthine oxidase form of Moco cannot. Alternatively, supplementation with the 

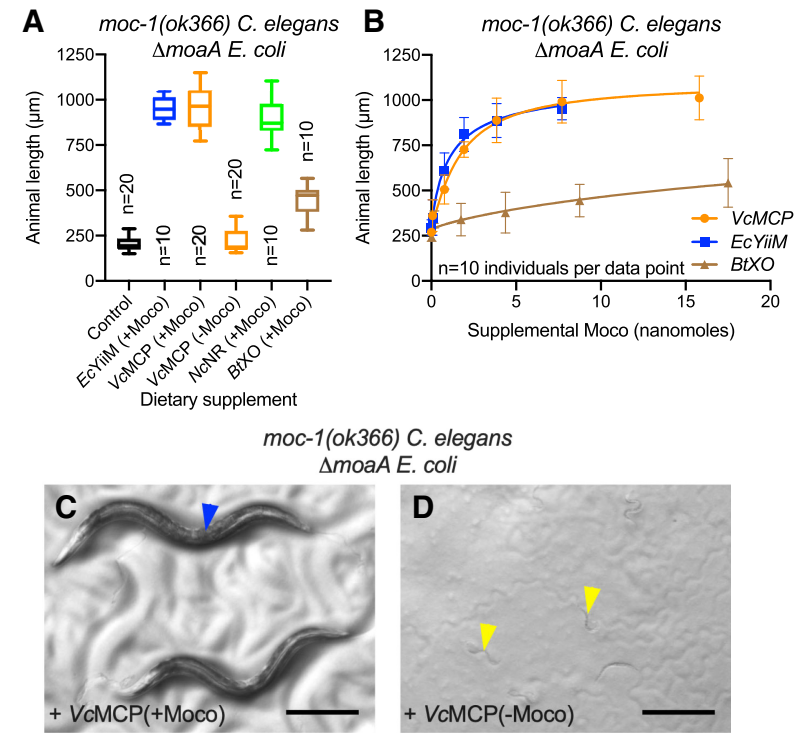

Figure 2. C. elegans uses Moco from diverse Moco-containing proteins. (A) moc-1(ok366) mutant C. elegans were synchronized at the L1 stage and cultured for $72 \mathrm{~h}$ on $\triangle m o a A E$. coli supplemented with Moco bound to Escherichia coli YiiM (EcYiiM), Volvox carteri Moco carrier protein $(V c \mathrm{MCP})$, Neurospora crassa nitrate reductase $(N c N R)$, or bovine xanthine oxidase (BtXO), or equivalent amounts of $V c M C P$ purified from bacteria that cannot synthesize Moco (-Moco). EcYiiM, VcMCP, and NcNR (+Moco) each contained 7.7 nmol of Moco, while BtXO (+Moco) contained $8.8 \mathrm{nmol}$ of Moco. Box plots display the median, upper, and lower quartiles, while whiskers indicate minimum and maximum data points. Sample size $(n)$ is displayed for each experiment. (B) moc-1(ok366) mutant C. elegans were synchronized at the L1 stage and cultured on $\triangle$ moaA E. coli supplemented with variable amounts of Moco bound to EcYiiM (0.0077, $0.077,0.77,1.93,3.85$, or $7.7 \mathrm{nmol}$ of Moco, blue), VcMCP, $(0.0077$, $0.077,0.77,1.93,3.85,7.7$, or $15.8 \mathrm{nmol}$ of Moco, orange) or BtXO $(0.018,0.18,1.8,4.38,8.75$, or $17.5 \mathrm{nmol}$ of Moco, brown). For each experiment, animals were allowed to develop for $72 \mathrm{~h}$ and animal lengths were determined. Mean and standard deviation are displayed for each data point. Sample size $(n)$ was 10 individuals assayed for each data point. $(C, D)$ Representative images of moc-1(ok366) C. elegans cultured for $72 \mathrm{~h}$ on $\triangle$ moaA E. coli supplemented with $7.7 \mathrm{nmol}$ of Moco bound to VcMCP $(C)$ or equivalent amounts of apo-VcMCP $(-\mathrm{Moco})(D)$. Blue arrowhead indicates a fertile adult, while yellow arrowheads denote animals arrested early in larval development. Scale bar, $250 \mu \mathrm{m}$.

sulfite oxidase form of Moco may result in the partial conversion, via Moco sulfurase (encoded by C. elegans mocs-1), of that supplemental Moco into the xanthine oxidase form (Fig. 1A). Thus, by providing the sulfite oxidase form of Moco we may be providing both forms of eukaryotic Moco making it a more effective treatment for complete Moco deficiency in C. elegans. Supplementation with the xanthine oxidase form of Moco would likely not result in synthesis of the sulfite oxidase form of Moco as there is no known enzyme that desulfurates the xanthine oxidase form of Moco.

To further demonstrate that the growth of C. elegans moc-1 mutant animals was conferred by supplementation of the Moco prosthetic group and not by the supplemental purified proteins, we purified apo- $V c M C P$ from bacteria unable to synthesize Moco. Supplemental apo$V c M C P$ did not support the growth of moc-1 mutant C. elegans fed $\triangle$ moaA mutant E. coli (Fig. 2A,C,D). Taken together these data demonstrate that the animal $C$. elegans is able to acquire and harvest the Moco prosthetic group when it is provided as a dietary supplement in complex with Moco-binding proteins. These proteins have diverse structures and functions and originate from both prokaryotes and eukaryotes. As such, the acquisition of protein-bound Moco by C. elegans is not specific to certain Moco-binding proteins and may reflect a general strategy for acquisition of functional Moco from the animals' diet or microbiome. The remaining experiments were all performed with supplemental Moco bound to $V c M C P$ due to its well-characterized role in Moco binding and our established protocols for its production (Witte et al. 1998; Hercher et al. 2020b).

One model for the rescue of C. elegans Moco deficiency is that supplemental protein-bound Moco is directly ingested by C. elegans. Alternatively, the protein-bound Moco may first be taken up by E. coli, which may process the Moco to then be ingested by C. elegans. To distinguish between these models, we grew $\triangle$ moaA mutant $E$. coli in lysogeny broth (LB) supplemented with Moco bound to $V c M C P$. This $\triangle m o a A$ E. coli was then separated from the culture medium by centrifugation, washed extensively, and fed to moc-1 mutant C. elegans ("diet B" in Fig. 3). Although cultured with Moco bound to VcMCP, the washed $\triangle$ moaA E. coli in diet B did not support growth of moc-1 mutant animals. Importantly, the supernatant medium from the same culture supported the growth of moc-1 mutant C. elegans grown on a lawn of $\triangle m o a A$ E. coli grown separately in LB alone ("diet A" in Fig. 3B). While we cannot completely exclude an active role for bacteria in the transfer of Moco to C. elegans under natural conditions, these data suggest that supplemental protein-bound Moco does not pass through a bacterial intermediate before being acquired by C. elegans (Fig. 3B).

\section{Moco bound to protein is stable}

The instability and oxygen sensitivity of Moco has limited cell biological studies of Moco transport and precluded it from therapeutic consideration (Schwarz 2016). The VcMCP-bound Moco used in "diet A" (Fig. 3) was incubated overnight at $37^{\circ} \mathrm{C}$ and still retained its activity and bioavailability, suggesting remarkable stability. To biochemically demonstrate the stability of Moco bound to protein, we measured the ability of mature Moco to stay in complex with $V c M C P, E c Y i i M, N c N R$, and BtXO over time (Fig. 4). Free Moco is highly unstable; however, it can be oxidized to "form A," a stable and fluorescent Moco derivative that is quantifiable via HPLC (Johnson et al. 1980; Hercher et al. 2020a). Using measurements of form A and protein concentration, we first determined the initial Moco occupancy of purified VcMCP $(22 \%)$ as well as $E c$ YiiM $(4 \%), N c$ NR $(50 \%)$, and $B t X O(50 \%)$ (Fig. $4 \mathrm{~A}, \mathrm{~B})$. We then assessed the stability of each purified Moco:protein complex by determining Moco retention over time at different temperatures (Fig. 4C-F). All four Moco:protein complexes were remarkably stable, showing no significant protein degradation and retaining between $43 \%$ and $83 \%$ of their original Moco content after $96 \mathrm{~h}$ of incubation at ambient temperature (Fig. 4C-F). This stability is surprising and suggests purification of protein-bound Moco as a new strategy for the production and delivery of therapeutically active Moco to treat MoCD. 


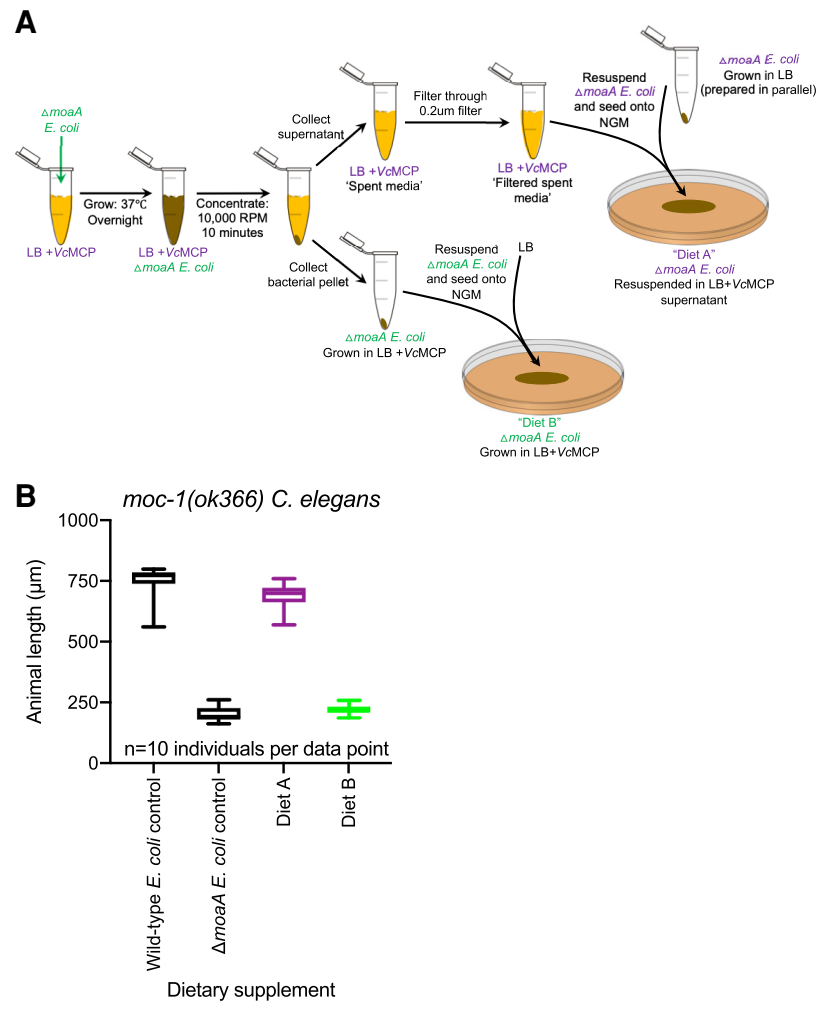

Figure 3. Protein-bound Moco is directly ingested by C. elegans. $(A)$

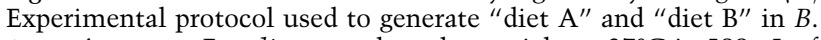
$\triangle$ moaA mutant $E$. coli were cultured overnight at $37^{\circ} \mathrm{C}$ in $500 \mu \mathrm{L}$ of LB supplemented with $39 \mathrm{nmol}$ of VcMCP-bound Moco. Bacterial cells were then concentrated, and the supernatant was removed for use in "diet A." Bacterial cells were washed, resuspended in LB, and seeded onto NGM to be fed to moc-1(ok366) C. elegans ("diet B"). The supernatant from this culture (spent LB + VcMCP media) was filtered $(0.20-\mu \mathrm{m}$ filter, Corning) to remove remaining bacterial cells and used to resuspend a separate concentrated culture of $\triangle m o a A$ mutant $E$. coli that was grown only in LB. This was then seeded onto NGM to be fed to moc-1(ok366) C. elegans ("diet A"). (B) moc-1 (ok366) mutant C. elegans were synchronized at the L1 stage and cultured on wild-type E. coli, $\triangle$ moaA E. coli, "diet A," or "diet B" (see A for $\operatorname{diet} \mathrm{A}$ and $\mathrm{B}$ descriptions). For each experiment, animals were allowed to develop for $48 \mathrm{~h}$. Box plots display the median, upper, and lower quartiles while whiskers indicate minimum and maximum data points. Sample size $(n)$ was 10 individuals assayed for each experiment.

Bioavailability of recombinant protein-bound Moco does not depend on known Moco biosynthetic enzymes in E. coli or C. elegans

We tested whether the Moco biosynthetic enzymes are necessary for the harvesting or transport of supplemental protein-bound Moco using mutants in the dietary E. coli. We tested moc-1 mutant C. elegans growth on wild-type bacteria, or mutant bacteria lacking the genes necessary for Moco biosynthesis. moc-1 mutant animals were grown on mutant $E$. coli with and without supplemental Moco bound to VcMCP. moc-1 mutant C. elegans grew well on wild-type E. coli but displayed larval arrest on all 10 E. coli mutants defective in Moco biosynthesis (Supplemental Fig. S1). Supplemental Moco bound to VcMCP supported growth and development of moc-1 mutant $C$. elegans on all 10 of the Moco biosynthetic mutant $E$. coli, demonstrating that none of these $E$. coli genes were necessary for bioavailability of supplemental proteinbound Moco (Supplemental Fig. S1).

Alternatively, we speculated that the Moco biosynthetic machinery of $C$. elegans might play a role in the bioavailability of supplemental protein-bound Moco. To test this, we used established C. elegans mutants in the Moco biosynthetic pathway (Fig. 1A). Each of these C. elegans moc mutants was cultured on wild-type $E$. coli, $\triangle$ moaA E. coli, or $\triangle$ moaA E. coli supplemented with Moco bound to $V c M C P$. All of the moc mutant animals grew well on wild-type bacteria and arrested growth on $\triangle$ moaA E. coli (Supplemental Fig. S2A-E). Each C. elegans moc mutant displayed dramatically improved growth on $\triangle m o a A$ E. coli when their diet was supplemented with Moco bound to VcMCP (Supplemental Fig. S2A-E). These results demonstrate that moc-5, moc-4, moc-3, moc-2, and moc-1 are not required for the bioavailability of supplemental protein-bound Moco. Thus, the machinery that facilitates Moco transport is distinct from the canonical Moco biosynthetic pathway.
A
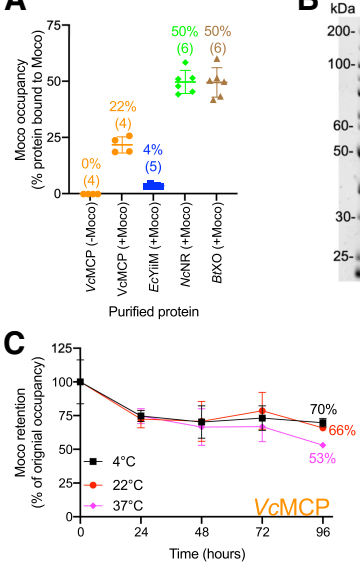

E

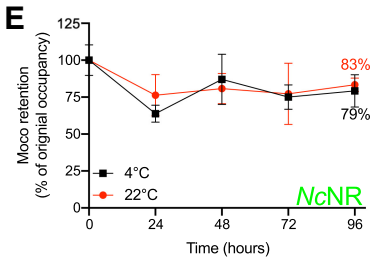

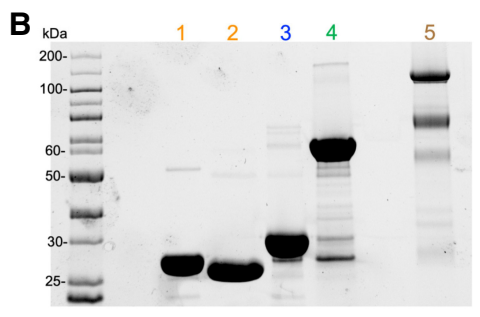

D

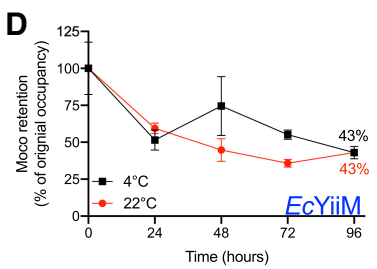

$\mathbf{F}$

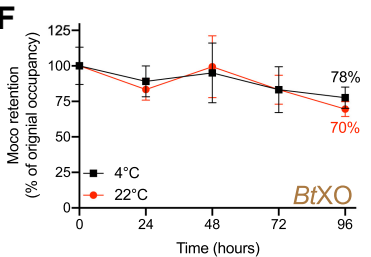

Figure 4. Stability of protein-bound Moco. $(A)$ Moco occupancies for purified $E c$ YiiM, $V c M C P, N c N R$, and $B t \mathrm{XO}$ were determined by measurements of the Moco derivative form $A$ and protein concentration. Moco occupancy is the percentage of protein molecules that are bound by a Moco prosthetic group. Moco occupancy was determined for $V c M C P$ purified from Moco-producing (+Moco) and Moco-deficient (-Moco) E. coli. The sample size $(n)$ is displayed for each protein and each data point is individually presented with the mean and standard deviation. $(B)$ Protein gel demonstrating the purity of $V c M C P$ purified from Moco-deficient E. coli (1), VcMCP (2), EcYiiM (3), and $N c N R$ purified from Moco-producing E. coli (4), and BtXO purified from bovine milk (5). The gel displays all protein using the TGX stain-free system (Bio-Rad). $(C-F)$ The amount of stable Moco retained by $V c M C P(C), E c Y i i M(D), N c N R(E)$, and $B t \mathrm{XO}(F)$ was determined over $96 \mathrm{~h}$ at $4^{\circ} \mathrm{C}$ (black) or $22^{\circ} \mathrm{C}$ (red). Moco retention of $V c M C P$ was also assessed at $37^{\circ} \mathrm{C}$ (pink). The $Y$-axis displays the Moco retention as a percentage of the original Moco occupancies (time 0 ) presented in $A$. The sample size $(n)$ is three to six replicates per protein and time point. The mean and standard deviation are displayed. 
Supplemental protein-bound Moco supports the activity of C. elegans SUOX-1

The lethality associated with Moco deficiency in C. elegans and humans is due to inactivity of sulfite oxidase (SUOX-1), a mitochondrial Moco-requiring enzyme that oxidizes the lethal toxin sulfite to sulfate. Like Moco biosynthesis, sulfite oxidase is essential in both C. elegans and humans (Mudd et al. 1967; Warnhoff and Ruvkun 2019). Thus, to rescue development of otherwise Mocodeficient C. elegans, supplemental protein-bound Moco must be incorporated into and support the activity of $C$. elegans SUOX-1. To demonstrate this, we used the hypomorphic suox-1 allele gk738847 (D391N) (Thompson et al. 2013). Aspartic acid 391 of sulfite oxidase is highly conserved and is present in C. elegans and humans. The SUOX-1 D391N amino acid substitution causes partial SUOX-1 loss of function that is enhanced when dietary Moco is absent. Growing suox-1(gk738847) mutant $C$. elegans on Moco-deficient E. coli causes a severe developmental delay compared with its growth on wild-type Moco-producing E coli (Supplemental Fig. S2F; Warnhoff and Ruvkun 2019). Importantly, suox-1(gk738847) mutant animals are wild type for their endogenous Moco biosynthetic pathway and are able to synthesize Moco de novo. This result shows that suox-1(gk738847) mutant C. elegans depend on both endogenous Moco biosynthesis as well as dietary sources of Moco to fully support the activity of mutant SUOX-1 D391N protein.

We hypothesized that supplemental Moco bound by $V c M C P$ would improve the viability of suox-1 $(g k 738847)$ animals grown on $\triangle m o a A E$. coli. To test this, we cultured suox-1(gk738847) mutant animals on wild-type E. coli, $\triangle m o a A E$. coli, and $\triangle m o a A E$. coli supplemented with Moco bound to VcMCP. Consistent with our rescue of $C$. elegans Moco deficiency, supplemental protein-bound Moco improved the growth of suox-1(gk738847) animals grown on Moco-deficient E. coli (Supplemental Fig. S2F). These results suggest that exogenous protein-bound Moco is absorbed, harvested, distributed to requisite cells, and reinserted into the C. elegans SUOX-1 enzyme. Uncovering the cellular mechanisms that facilitate these processes is an important goal of future research. Pathways for absorption and distribution of heme and vitamin B12 serve as useful paradigms for the possible mechanisms of Moco transport. These metal-containing prosthetic groups require chaperones (Dieckgraefe et al. 1988; Chen et al. 2011), receptors (Moestrup et al. 1998), and transporters (Rajagopal et al. 2008) to facilitate their movement among cells and tissues. We anticipate similar systems will coordinate the transport of Moco in C. elegans and humans.

Our data demonstrate the ability of an essential proteinpackaged prosthetic group to cross cell membranes. We show that this transfer occurs naturally between multiple organisms and among the cells and tissues of a single organism. Because Moco biosynthesis and utilization is as ancient as the last universal common ancestor, the unknown Moco transport pathway is likely to be general to all animals. Furthermore, roughly $70 \%$ of bacterial genomes encode Moco biosynthetic enzymes making the intestinal microbiome a potential reservoir for this cofactor (Zhang and Gladyshev 2008). Similarly, the human diet might also be a source of exogenous protein-bound Moco as most plants and animals synthesize and use Moco. Our results with the nematode C. elegans may stimulate future exploration of the therapeutic potential of protein-bound Moco (from dietary, microbiome, or recombinant sources) for the treatment of MoCD.

\begin{abstract}
Materials and methods
General methods and strains

C. elegans strains were cultured using established protocols (Brenner 1974). The wild-type strain of C. elegans was Bristol N2. C. elegans mutant strains used in this work are listed here. LGI: GR2253 moc-4(ok2571). LGIV: MH3266 moc-3(ku300). LGV: GR2255 moc-2(mg595). LGX: GR2254 moc-1(ok366), GR2256 moc-5(mg589), and GR2269 suox-1 (gk738847).

E. coli strains were cultured using standard methods. The wild-type strain of E. coli was BW25113, the parental strain of the Keio E. coli knockout collection (Baba et al. 2006). The E. coli mutants used in this work were JW0764 $\left(\Delta m o a A:: \operatorname{Kan}^{\mathrm{r}}\right)$, KJW1 $(\Delta m o g)$, KJW2 $(\Delta m o a A)$, KJW3 $(\Delta m o a C)$,

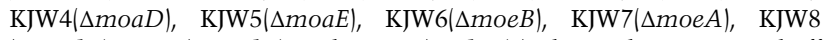
$(\Delta \bmod A), \mathrm{KJW} 9(\Delta \bmod C)$, and KJW10( $\triangle \mathrm{ydaV})($ Baba et al. 2006; Warnhoff and Ruvkun 2019). Strains KJW1-KJW10 were only used to produce the data in Supplemental Figure S1. JW0764 was used in all other experiments with $\triangle$ moaA E. coli.
\end{abstract}

\section{C. elegans growth assays}

C. elegans were synchronized at the first stage of larval development (L1), then cultured on NGM seeded with wild-type or mutant E. coli. For some experiments, growth conditions were supplemented with various forms and amounts of protein-bound Moco. For experiments with supplemental protein-bound Moco, we report the total amount of Moco added to the C. elegans growth conditions and assume equal protein diffusion through the solid agarose media during the experiments. C. elegans animals were allowed to grow and develop for 48 or $72 \mathrm{~h}$ (specified in the figure legends) at $20^{\circ} \mathrm{C}$. Sample size $(n)$ is individual animals measured and is reported in the figures and figure legends.

Live animals were imaged using an Axio Zoom.V16 microscope (Zeiss) equipped with an ORCA-Flash4.0 digital camera (Hamamatsu). Images were captured using Zen software (Zeiss) and processed with ImageJ (NIH). Animal length was measured from the tip of the head to the end of the tail. The median and upper and lower quartiles were calculated using GraphPad Prism software.

\section{Purification and characterization of Moco-binding proteins}

Moco-binding proteins were expressed and purified using standard methods (Hercher et al. 2020a). The full-length yiiM coding sequence was amplified from Escherichia coli DH5a (EcYiiM) and the full-length Moco carrier protein coding sequence from Volvox carteri (VcMCP) was synthesized and codon optimized for E. coli (Hercher et al. 2020b). The coding sequence for Neurospora crassa nitrate reductase ( $N c \mathrm{NR}$ ) was shortened to include only the Moco-binding and dimerization region (amino acids 113-592). The coding sequences for $E c Y i i M, V c M C P$, and $N c N R$ were inserted into the pONE-CP plasmid, producing proteins fused to a C-terminal Streptavidin tag. Streptavidin-tagged proteins were expressed using the E. coli strain TP1000, which accumulates Moco due to a deletion in the Mob operon (Palmer et al. 1996). As a negative control, VcMCP was also purified from the E. coli strain RK5204, which is unable to produce Moco (Stewart and MacGregor 1982). Bovine xanthine oxidase (BtXO) was purchased from Sigma-Aldrich (X1875, batch SLCB1289).

Protein concentrations were determined using absorption at $280 \mathrm{~nm}$ and the Pierce BCA protein assay (Thermo Scientific). Absorption was measured using a Multiskan GO microplate spectrophotometer (Thermo Scientific). Quantification of Moco content of the proteins was conducted using HPLC-based measurements of form $\mathrm{A}$, a stable and fluorescent Moco oxidation product (Hercher et al. 2020a). Stability of protein-bound Moco was assessed by incubating the Moco:protein complexes for $96 \mathrm{~h}$ at various temperatures $\left(4^{\circ} \mathrm{C}, 22^{\circ} \mathrm{C}\right.$, and $\left.37^{\circ} \mathrm{C}\right)$. In 24 -h intervals, protein samples were centrifuged at $4^{\circ} \mathrm{C}$ to remove precipitated protein, and protein concentration and Moco content were determined as described above. 


\section{Acknowledgments}

We thank the Caenorhabditis Genetics Center (CGC) for providing C. elegans strains, and the National BioResource Project (NIG, Japan) for providing the Keio E. coli knockout collection. We thank Dr. Jörn Krausze for help in crystal structure-guided determination of the stereochemistry of Moco intermediates in Figure 1A. This work was funded by a National Institutes of Health grant (5R01GM044619-26) to G.R., a Deutsche Forschungsgemeinschaft grant (GRK2223/1) to R.R.M., and a Damon Runyon Fellowship (DRG-2293-17) to K.W.

Author contributions: K.W., T.W.H., R.R.M., and G.R. conceived of the experiments, interpreted data, and wrote the manuscript. K.W. and T.W.H. performed experiments.

\section{References}

Baba T, Ara T, Hasegawa M, Takai Y, Okumura Y, Baba M, Datsenko KA, Tomita M, Wanner BL, Mori H. 2006. Construction of Escherichia coli $\mathrm{K}-12$ in-frame, single-gene knockout mutants: the Keio collection. Mol Syst Biol 2: 1-11. doi:10.1038/msb4100050

Bittner F, Oreb M, Mendel RR. 2001. ABA3 is a molybdenum cofactor sulfurase required for activation of aldehyde oxidase and xanthine dehydrogenase in Arabidopsis thaliana. I Biol Chem 276: 40381-40384. doi:10.1074/ibc.C100472200

Brenner S. 1974. The genetics of Caenorhabditis elegans. Genetics 77: 71-94.

Chen C, Samuel TK, Sinclair J, Dailey HA, Hamza I. 2011. An intercellular heme-trafficking protein delivers maternal heme to the embryo during development in C. elegans. Cell 145: 720-731. doi:10.1016/j.cell.2011 .04 .025

Dieckgraefe BK, Seetharam B, Banaszak L, Leykam JF, Alpers DH. 1988. Isolation and structural characterization of a cDNA clone encoding rat gastric intrinsic factor. Proc Natl Acad Sci 85: 46-50. doi:10 $.1073 /$ pnas.85.1.46

Enroth C, Eger BT, Okamoto K, Nishino T, Nishino T, Pai EF. 2000. Crystal structures of bovine milk xanthine dehydrogenase and xanthine oxidase: structure-based mechanism of conversion. Proc Natl Acad Sci 97: 10723-10728. doi:10.1073/pnas.97.20.10723

Fischer K, Barbier GG, Hecht H-J, Mendel RR, Campbell WH, Schwarz G. 2005. Structural basis of eukaryotic nitrate reduction: crystal structures of the nitrate reductase active site. Plant Cell 17: 1167-1179. doi:10.1105/tpc. 104.029694

Hercher TW, Krausze J, Hoffmeister S, Zwerschke D, Lindel T, Blankenfeldt W, Mendel RR, Kruse T. 2020a. Insights into the Cnx1E catalyzed MPT-AMP hydrolysis. Biosci Rep 40: BSR20191806. doi:10.1042/ BSR20191806

Hercher TW, Krausze J, Yang J, Kirk ML, Kruse T. 2020b. Identification and characterization of the Volvox carteri Moco carrier protein. Biosci Rep 40: BSR20202351. doi:10.1042/BSR20202351

Hille R, Hall J, Basu P. 2014. The mononuclear molybdenum enzymes. Chem Rev 114: 3963-4038. doi:10.1021/cr400443z

Huijmans JGM, Schot R, de Klerk JBC, Williams M, de Coo RFM, Duran M, Verheijen FW, van Slegtenhorst M, Mancini GMS. 2017. Molybdenum cofactor deficiency: identification of a patient with homozygote mutation in the MOCS3 gene. Am J Med Genet 173: 1601-1606. doi:10.1002/ajmg.a.38240

Johnson JL, Hainline BE, Rajagopalan KV. 1980. Characterization of the molybdenum cofactor of sulfite oxidase, xanthine, oxidase, and nitrate reductase. identification of a pteridine as a structural component. $I$ Biol Chem 255: 1783-1786.

Kozmin SG, Leroy P, Pavlov YI, Schaaper RM. 2008. Ycbx and yiiM, two novel determinants for resistance of Escherichia coli to N-hydroxylated base analogues. Mol Microbiol 68: 51-65. doi:10.1111/j.1365-2958 .2008.06128.x

MacGregor CH. 1975. Synthesis of nitrate reductase components in chlorate-resistant mutants of Escherichia coli. J Bacteriol 121: 1117-1121. doi:10.1128/JB.121.3.1117-1121.1975
Mendel RR. 1983. Release of molybdenum co-factor from nitrate reductase and xanthine oxidase by heat treatment. Phytochemistry 22: 817-819. doi:10.1016/0031-9422/83/85004-3

Mendel RR. 2013. The molybdenum cofactor. J Biol Chem 288: 1316513172. doi:10.1074/jbc.R113.455311

Moestrup SK, Kozyraki R, Kristiansen M, Kaysen JH, Rasmussen $\mathrm{HH}_{,}$ Brault D, Pontillon F, Goda FO, Christensen EI, Hammond TG, et al. 1998. The intrinsic factor-vitamin $B_{12}$ receptor and target of teratogenic antibodies is a megalin-binding peripheral membrane protein with homology to developmental proteins. I Biol Chem 273: 5235-5242. doi:10.1074/jbc.273.9.5235

Mudd SH, Irreverre F, Laster L. 1967. Sulfite oxidase deficiency in man: demonstration of the enzymatic defect. Science 156: 1599-1602. doi:10.1126/science.156.3782.1599

Namgung B, Kim J-H, Song WS, Yoon S-I. 2018. Crystal structure of the hydroxylaminopurine resistance protein, YiiM, and its putative molybdenum cofactor-binding catalytic site. Sci Rep 8: 3304-3312. doi:10.1038/s41598-018-21660-y

Neumann M, Stöcklein W, Walburger A, Magalon A, Leimkühler S. 2007. Identification of a Rhodobacter capsulatus L-cysteine desulfurase that sulfurates the molybdenum cofactor when bound to XdhC and before its insertion into xanthine dehydrogenase. Biochemistry 46: 95869595. doi:10.1021/bi700630p

Palmer T, Santini CL, Iobbi-Nivol C, Eaves DJ, Boxer DH, Giordano G. 1996. Involvement of the narj and mob gene products in distinct steps in the biosynthesis of the molybdoenzyme nitrate reductase in Escherichia coli. Mol Microbiol 20: 875-884. doi:10.1111/j.1365-2958.1996 .tb02525.x

Rajagopal A, Rao AU, Amigo J, Tian M, Upadhyay SK, Hall C, Uhm S, Mathew MK, Fleming MD, Paw BH, et al. 2008. Haem homeostasis is regulated by the conserved and concerted functions of HRG-1 proteins. Nature 453: 1127-1131. doi:10.1038/nature06934

Rajagopalan KV, Johnson JL. 1992. The pterin molybdenum cofactors. I Biol Chem 267: 10199-10202.

Reiss J, Hahnewald R. 2011. Molybdenum cofactor deficiency: mutations in GPHN, MOCS1, and MOCS2. Hum Mutat 32: 10-18. doi:10.1002/ humu. 21390

Schwarz G. 2016. Molybdenum cofactor and human disease. Curr Opin Chem Biol 31: 179-187. doi:10.1016/j.cbpa.2016.03.016

Schwarz G, Mendel RR, Ribbe MW. 2009. Molybdenum cofactors, enzymes and pathways. Nature 460: 839-847. doi:10.1038/nature 08302

Stewart V, MacGregor CH. 1982. Nitrate reductase in Escherichia coli K12: involvement of chlC, chlE, and chlG loci. J Bacteriol 151: 788-799. doi:10.1128/JB.151.2.788-799.1982

Thompson O, Edgley M, Strasbourger P, Flibotte S, Ewing B, Adair R, Au V, Chaudhry I, Fernando L, Hutter H, et al. 2013. The million mutation project: a new approach to genetics in Caenorhabditis elegans. Genome Res 23: 1749-1762. doi:10.1101/gr.157651.113

Veldman A, Santamaria-Araujo JA, Sollazzo S, Pitt J, Gianello R, Yaplito-Lee J, Wong F, Ramsden CA, Reiss J, Cook I, et al. 2010. Successful treatment of molybdenum cofactor deficiency type A with cPMP. Pediatrics 125: e1249-e1254. doi:10.1542/peds.20092192

Warnhoff K, Ruvkun G. 2019. Molybdenum cofactor transfer from bacteria to nematode mediates sulfite detoxification. Nat Chem Biol 15: 480488. doi:10.1038/s41589-019-0249-y

Weiss MC, Sousa FL, Mrnjavac N, Neukirchen S, Roettger M, NelsonSathi S, Martin WF. 2016. The physiology and habitat of the last universal common ancestor. Nat Microbiol 1: 16116-8. doi:10.1038/nmi crobiol.2016.116

Witte CP, Igeño MI, Mendel R, Schwarz G, Fernández E. 1998. The Chlamydomonas reinhardtii MoCo carrier protein is multimeric and stabilizes molybdopterin cofactor in a molybdate charged form. FEBS Lett 431: 205-209. doi:10.1016/S0014-5793/98/00756-X

Zhang Y, Gladyshev VN. 2008. Molybdoproteomes and evolution of molybdenum utilization. I Mol Biol 379: 881-899. doi:10.1016/j.jmb .2008 .03 .051 


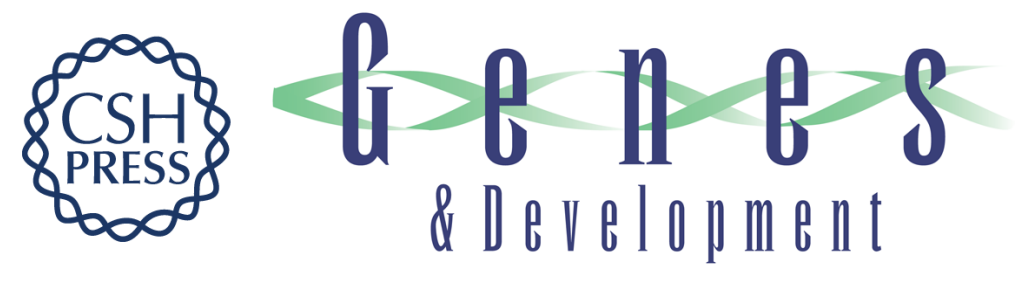

\section{Protein-bound molybdenum cofactor is bioavailable and rescues molybdenum cofactor-deficient $C$. elegans}

Kurt Warnhoff, Thomas W. Hercher, Ralf R. Mendel, et al.

Genes Dev. 2021, 35: originally published online January 14, 2021

Access the most recent version at doi:10.1101/gad.345579.120

\section{Supplemental http://genesdev.cshlp.org/content/suppl/2021/01/12/gad.345579.120.DC1 \\ Material}

Related Content

Learning from the worm: the effectiveness of protein-bound Moco to treat Moco deficiency

Aileen K. Sewell and Min Han

Genes Dev. February , 2021 35: 177-179

References This article cites 33 articles, 13 of which can be accessed free at:

http://genesdev.cshlp.org/content/35/3-4/212.full.html\#ref-list-1

Articles cited in:

http://genesdev.cshlp.org/content/35/3-4/212.full.html\#related-urls

Creative This article is distributed exclusively by Cold Spring Harbor Laboratory Press for the first Commons six months after the full-issue publication date (see

License http://genesdev.cshlp.org/site/misc/terms.xhtml). After six months, it is available under a Creative Commons License (Attribution-NonCommercial 4.0 International), as described at http://creativecommons.org/licenses/by-nc/4.0/.

Email Alerting Receive free email alerts when new articles cite this article - sign up in the box at the top Service right corner of the article or click here.

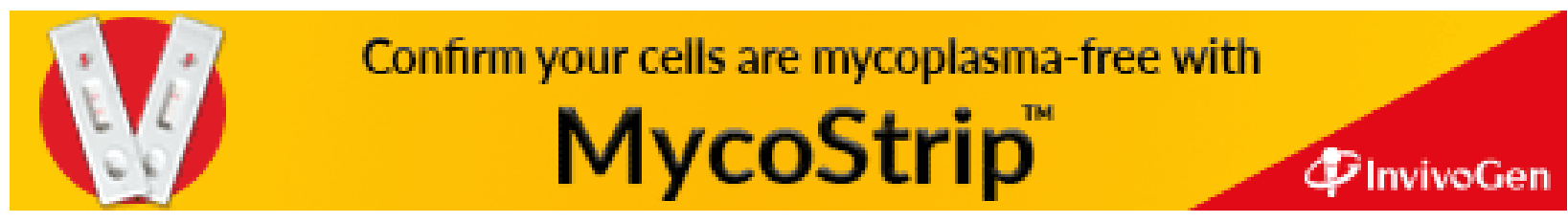

